\author{
Supplementary Information
}

\title{
Digital Microfluidic Thermal Control Chip based Multichannel Immunosensor for Non-invasive Detecting Acute Myocardial Infarction
}

Jienan Shen, ${ }^{[a][c][d]+}$ Liyuan Zhang, ${ }^{[e]+}$ Junjie Yuan, ${ }^{[b]+}$ Yongsheng Zhu, ${ }^{[a]}$ Hao Cheng, ${ }^{[a]}$ Yibo Zeng, ${ }^{[a]}$ Jiaqin Wang, ${ }^{[a]}$ Xueqiu You, ${ }^{[a]}$ Chaoyong Yang, ${ }^{[a]}$ Xiangmeng $Q u,{ }^{[b]^{*}}$ Hong Chen, ${ }^{[a][f]^{*}}$

[a] Pen-Tung Sah Institute of Micro-Nano Science and Technology, College of Chemistry and Chemical Engineering, School of Electronic Science and Engineering (National Model Microelectronics College), Xiamen University, Xiamen 361005, China

[b] School of Biomedical Engineering, Sun Yat-Sen University, Shenzhen 518107, China

[c] Hwa Mei Hospital, University of Chinese Academy of Sciences, Ningbo 315000, China

[d] Ningbo Institute of Life and Health Industry, University of Chinese Academy of Sciences, Ningbo 315000, China

[e] Harvard John A. Paulson School of Engineering and Applied Sciences, Harvard University, Cambridge, MA 02138, USA

[f] Jiujiang Research Institute of Xiamen University, Jiujiang 332000, China

$[+]$ These authors contributed equally to this work

Corresponding author:

Hong Chen, E-mail: hongc@xmu.edu.cn;

Xiangmeng Qu, E-mail: quxm5@mail.sysu.edu.cn.

\section{Table of contents}

Figure S1. Desing of the DMF holder and PCB connector

Figure S2. The volume variation of the droplets generated by manual pipetting

Figure S3. Design of the DMF thermal control chip

Figure S4. The specificity of detection three biomarkers

Figure S5. Manufacturing process of the digital microfluidic thermal control chip

Figure S6. The components of the DMF thermal control chip

Figure S7. Combined detection process of multiple AMI's biomarkers

Figure S8. The experiment of the human serum samples

Table S1. Recovery experiments of cTnI, Myo and CK-MB in human serum samples 


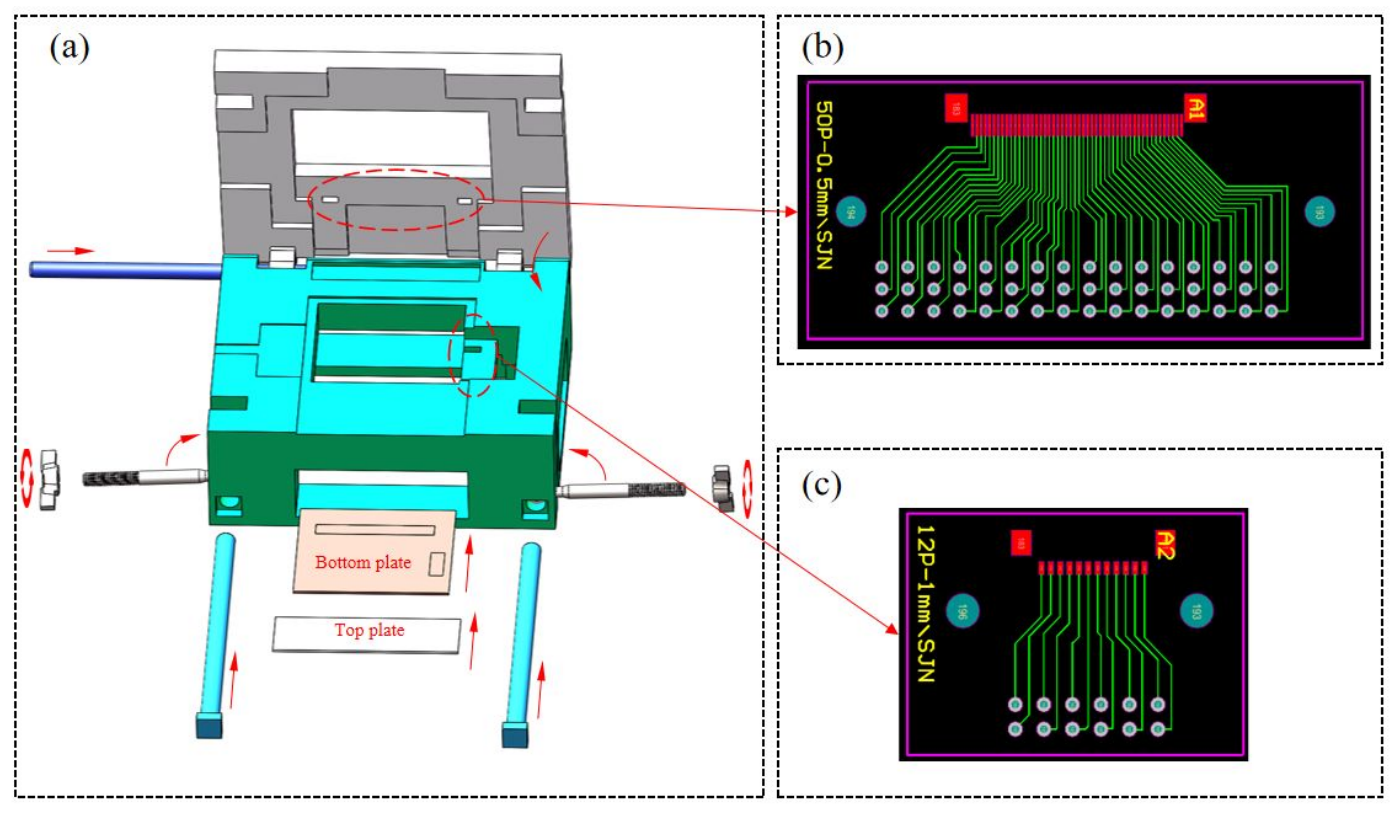

Figure S1. Desing of the DMF holder and PCB connector. (a) The 3D exploded view of the chip holder. (b) The design of the PCB connector for drive electrodes. (c) The design of the PCB connector for the heating wire. 


\section{Variation in droplet volume generated by manual pipetting}

Compared with the droplets generated by the DMF chip, the droplets were also generated by the traditional method (manual pipetting). As shown in Figure S2. Twenty droplets of approximately $1.7 \mu \mathrm{L}$ were generated by manual pipetting, and the $\mathrm{CV}$ of the droplets' volume determined by their weight was calculated to be $2.06 \%$.

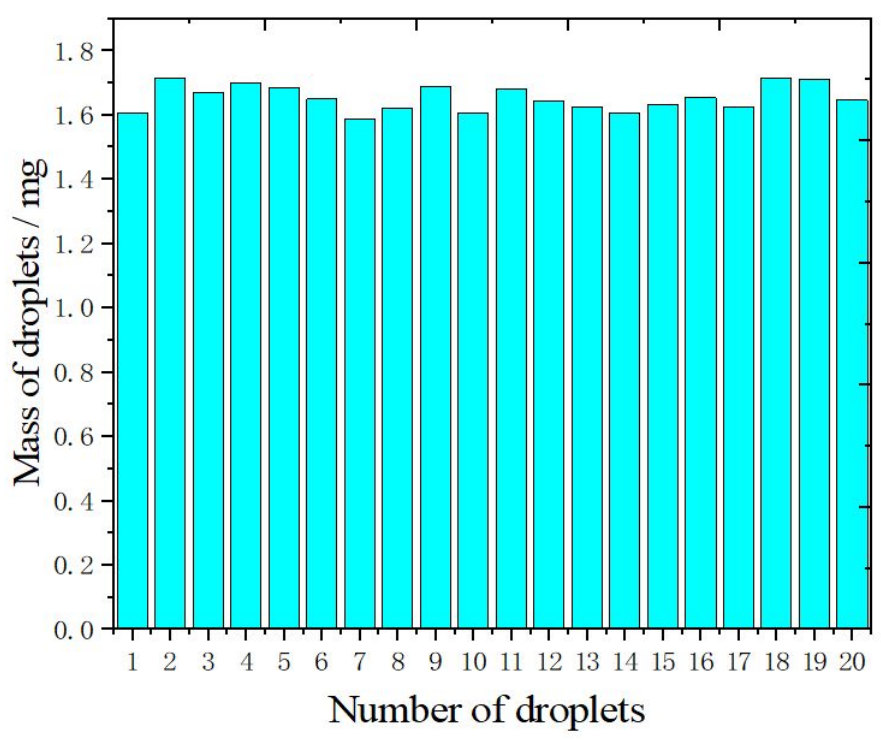

Figure S2. The volume variation of the droplets generated by manual pipetting. 


\section{Design of the DMF thermal control chip}

Figure S3 shows the design of the DMF chip integrated with heating components. In Figure S3a, the blue area represents the waiting area composed of 6 liquid storage electrodes, the black area represents the functional area composed of 39 driving electrodes, the yellow area represents the detection area composed of 3 driving electrodes, and the red area represents the serpentine heating wire on the back (also shown in Figure S3c). Figure S3b shows the DMF chip. Figure S3c and Figure S3d show the design and DMF plate of integrated heating components.
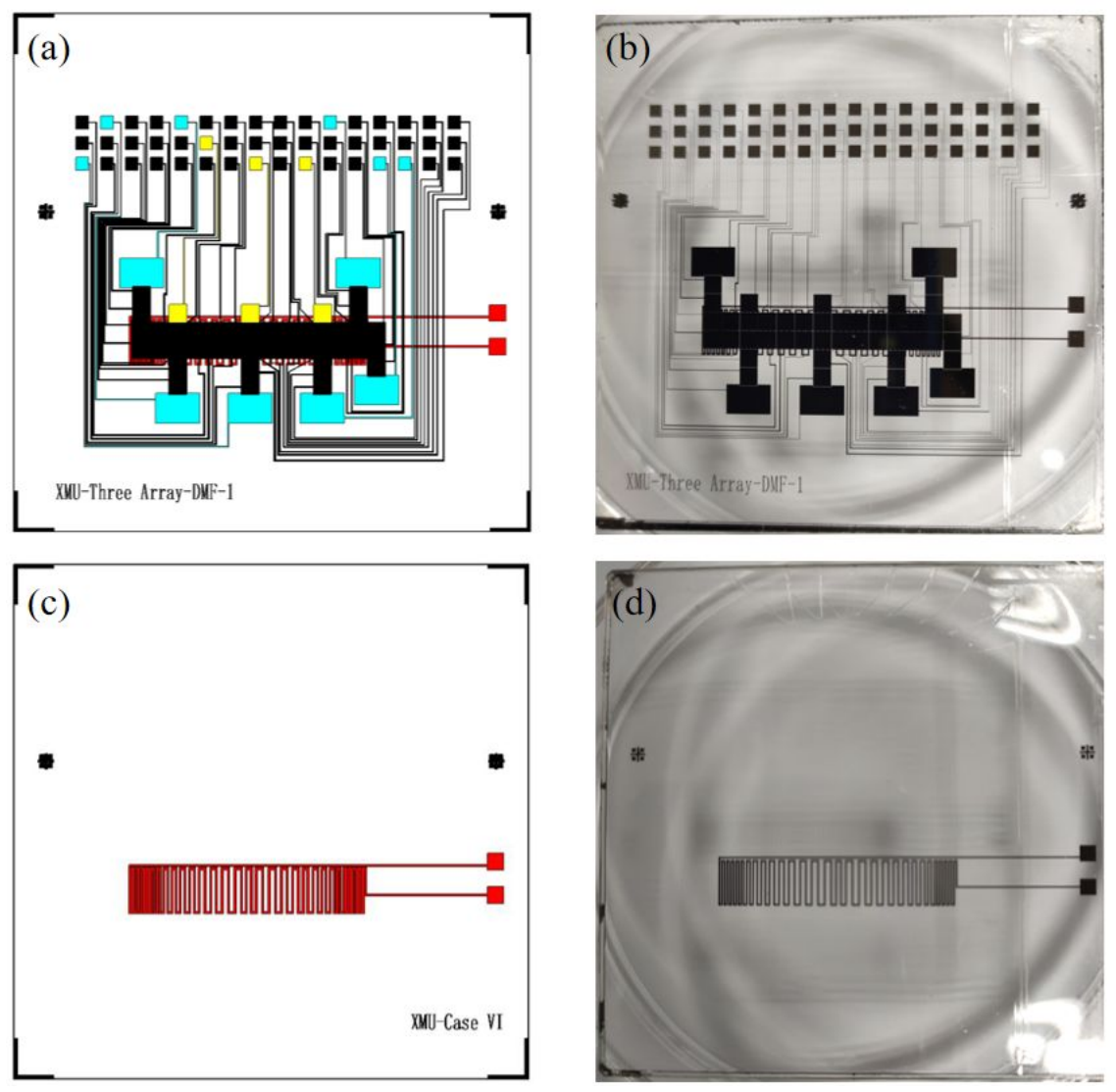

Figure S3. (a) Design of the DMF thermal control chip. (b) The final DMF chip. (c) The design of the heating component. (d) The DMF plate with heating components. 


\section{The specificity of detection three biomarkers}

Before combined detection on the DMF chip, the specificity of the three biomarkers was verified, and the result is shown in Figure S4. Figure S4a shows the fluorescence intensity of a sample containing only $1000 \mathrm{ng} / \mathrm{mL}$ Myo. Figure $\mathrm{S} 4 \mathrm{~b}$ shows the fluorescence intensity of a sample containing only $100 \mathrm{ng} / \mathrm{mL}$ CK-MB. Figure S4c shows the fluorescence intensity of a sample containing only $10 \mathrm{ng} / \mathrm{mL} \mathrm{cTnI}$.
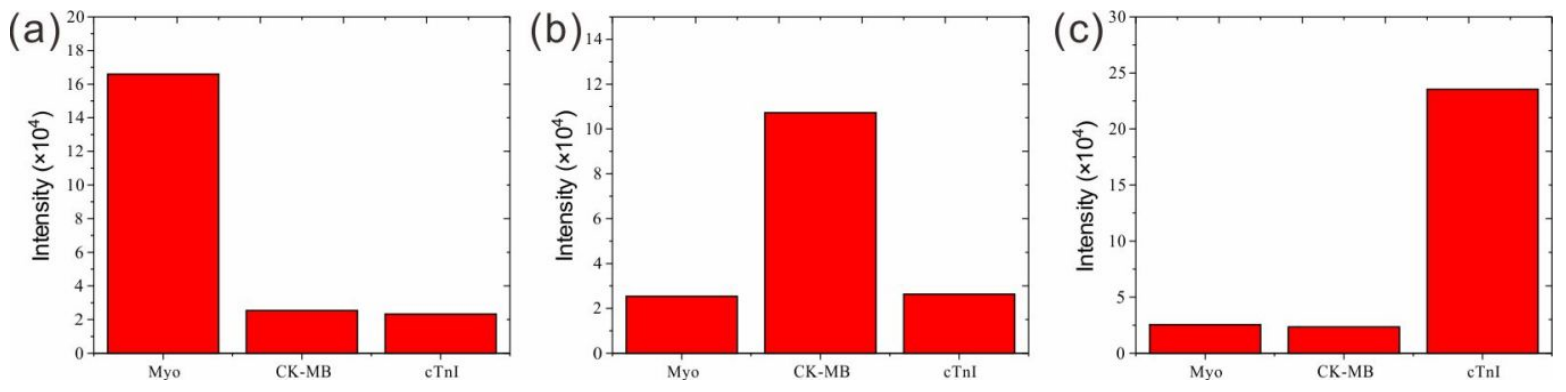

Figure S4. The selectivity between Myo, CK-MB and cTnI. (a) The fluorescence intensity of the sample containing only Myo. (b) The fluorescence intensity of the sample containing only CK-MB. (c) The fluorescence intensity of the sample containing only cTnI. 


\section{Fabrication process of DMF chip with integrated heating component}

Figure S5 shows the manufacturing flow chart for the DMF chip integrated with the heating component. The detailed process description is as follows:

a) A piranha solution (concentrated sulfuric acid:hydrogen peroxide $=3: 1$ ) was used to clean the glass substrate.

b) A chromium layer (400 $\mathrm{nm}$ in depth) was grown on the glass substrate by magnetron sputtering.

c) A photoresist (AZ5214E) was coated on the chromium layer, exposed and developed.

d) The exposed chromium was etched by etching solution.

e) After the etching was completed, the photoresist was removed by acetone.

f) A photoresist (SU-8 2015) was coated on the substrate, exposed and post-baked.

g) The substrate was turned over, and a photoresist (AZ5214E) was coated on the substrate, exposed and developed.

h) A platinum layer (200 $\mathrm{nm}$ in depth) was sputtered on the substrate by magnetron sputtering.

i) Acetone was used to eliminate the photoresist.

j) A photoresist (SU-8 2015) was coated on the substrate, exposed and post-baked.

k) The substrate was turned over and Teflon AF ( $200 \mathrm{~nm}$ in depth) was coated on the substrate.
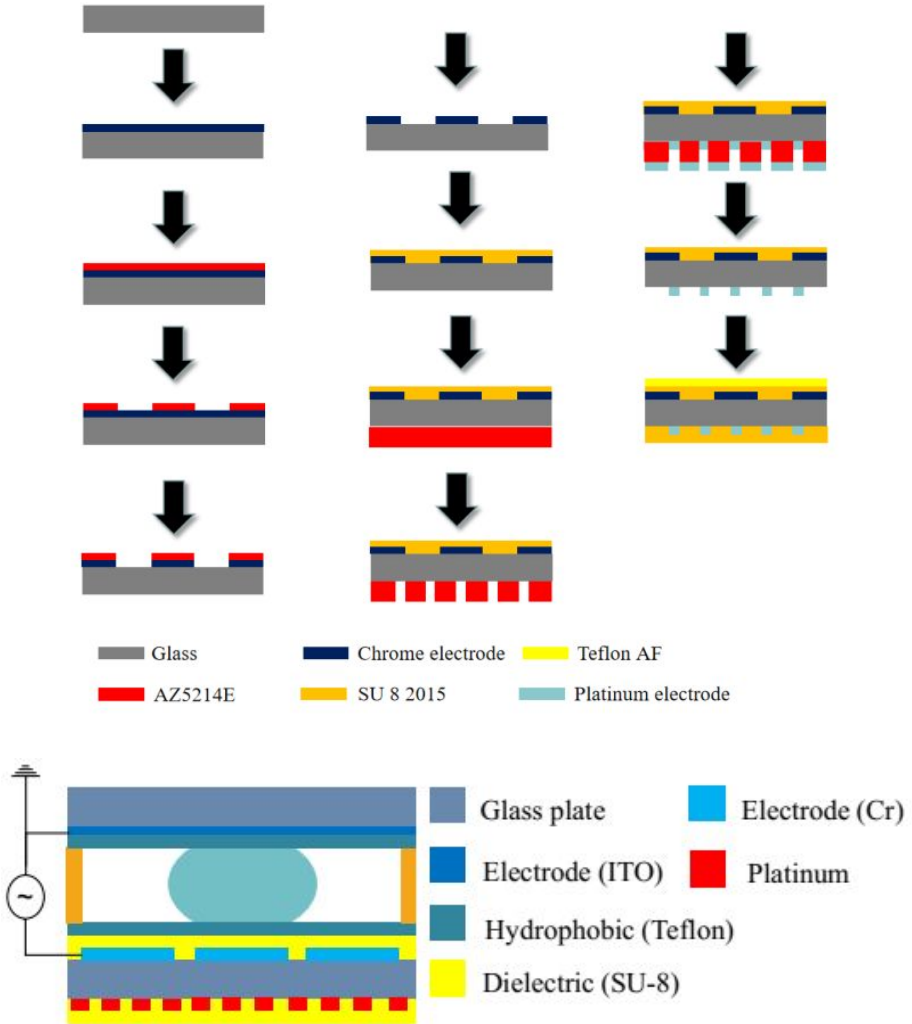

Figure S5. The manufacturing flow chart for the DMF chip, and the principle of EWOD. 
The components of the DMF thermal control chip.
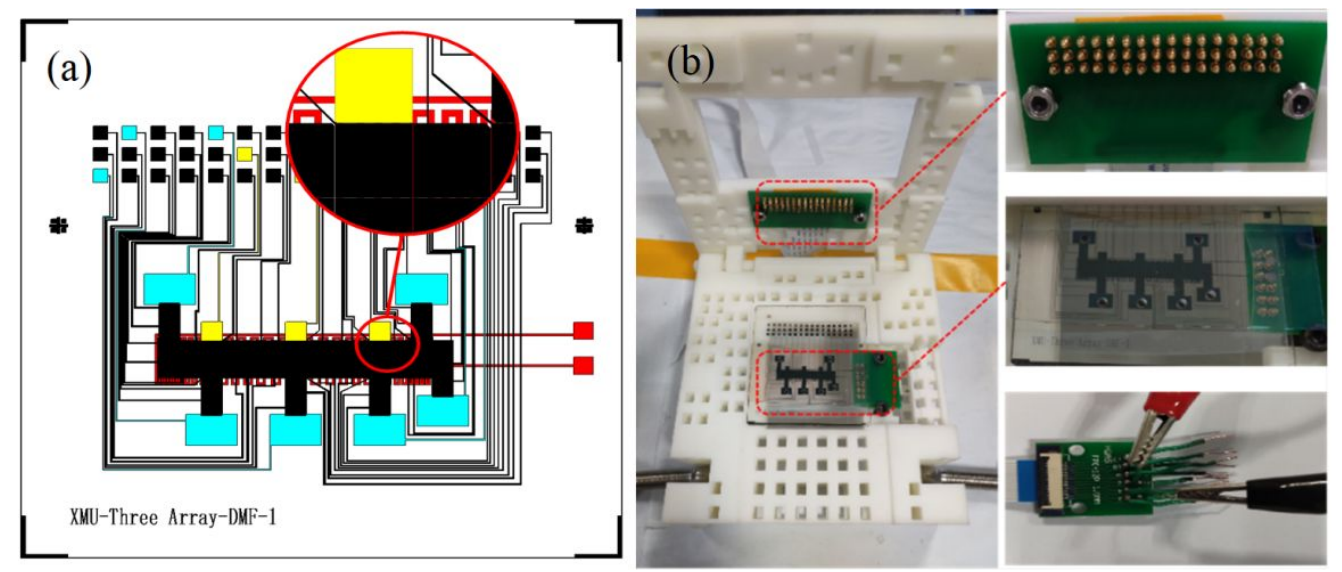

Figure S6. (a) The design of the DMF chip. (b) The images in the right column are (from top to bottom): a spring-mounted connector (pogo pin) for electrical connection with the DMF chip; a spring-mounted connector (pogo pin) of heating electrode for electrical connection with DC power; and an adapter for connecting the power supply and the heating cable. 


\section{Combined detection process of multiple biomarkers.}

(a)

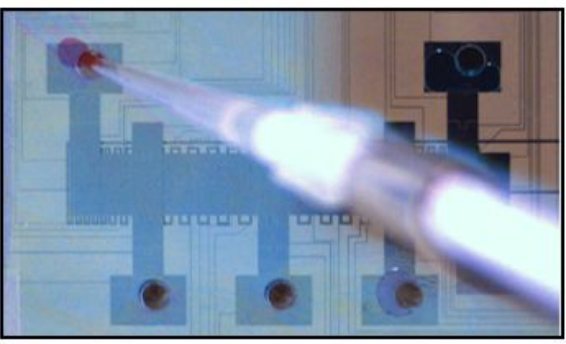

(b)

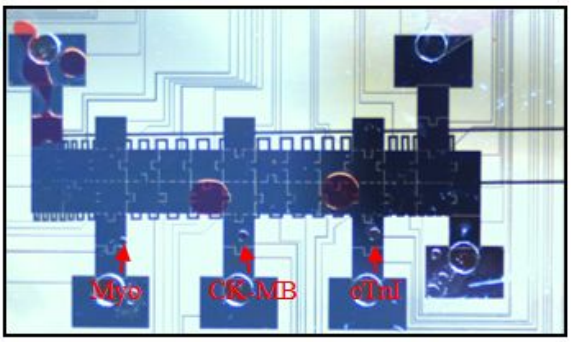

(c)

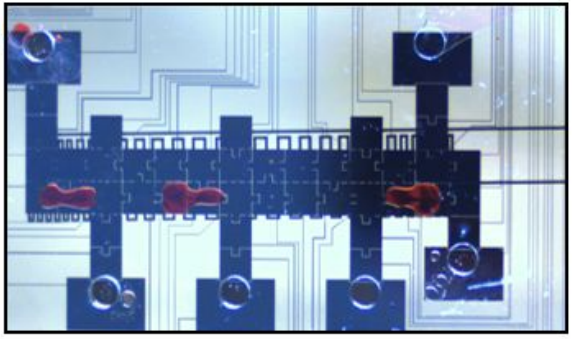

(d)

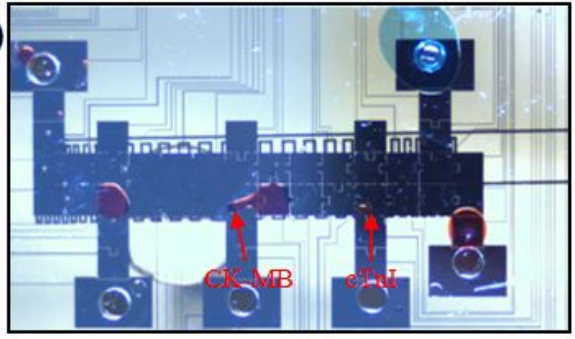

(e)

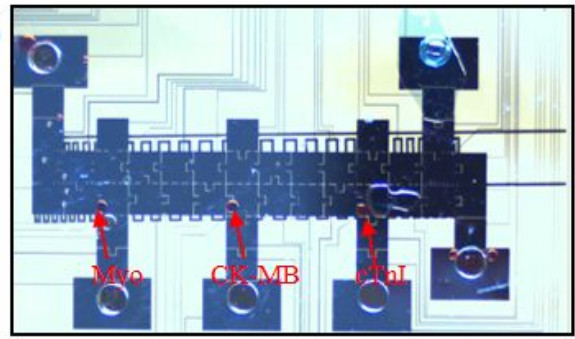

(f)

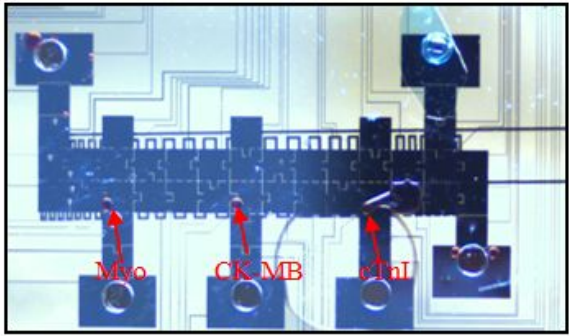

(g)

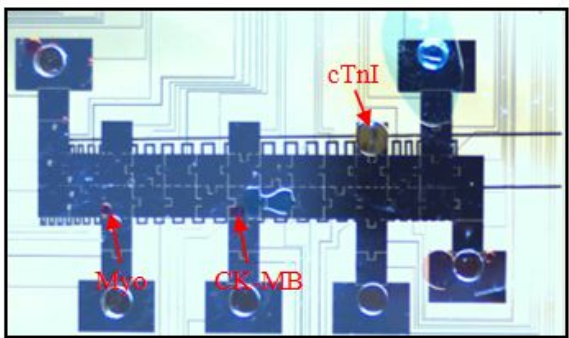

(h)

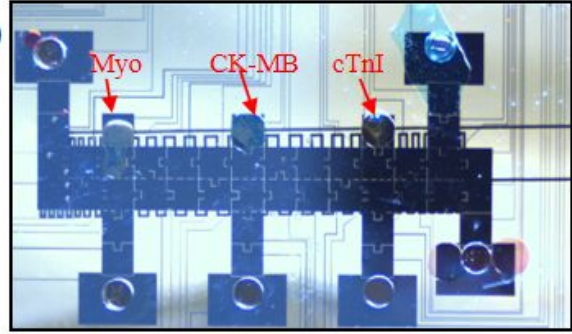

Figure S7. The combined detection processes. (a) Adding sample to the reservoir by pipetting. (b) Generating the sample droplets and merging with the corresponding magnetic beads. (c) Mixing and incubation. (d) Separating sample droplets and retaining the magnetic beads. (e) Dispensing washing buffer and mixing (taking cTnI as an example). (f) Separating of washing buffer and retaining the magnetic beads. (g) Merging with dilution buffer and moving to the detection area. (h) Waiting for detection. 
The experiment of the human serum samples.
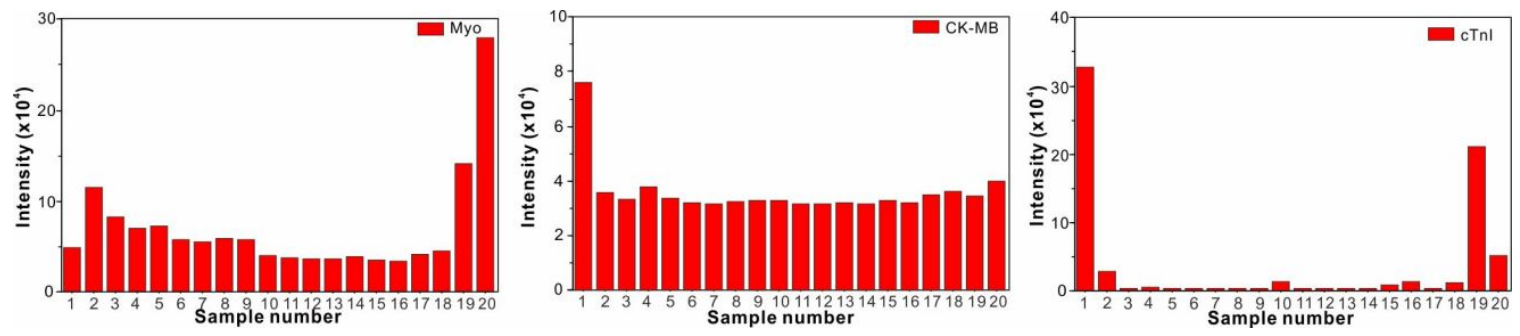

Figure S8. The fluorescence intensity of the human serum sample by using the multichannel-immunosensor detection.

Table S1. Recovery experiments of cTnI, Myo and CK-MB in human serum samples

\begin{tabular}{cccc}
\hline Target biomarker & $\begin{array}{c}\text { Added amount } \\
(\mathrm{ng} / \mathrm{ml})\end{array}$ & Detected (ng/ml) & Recoveries (\%) \\
\hline CTnI-1 & 0.15 & 0.1671 & 111.4 \\
CTnI-2 & 0.3 & 0.3170 & 105.7 \\
CTnI-3 & 1 & 1.1440 & 114.4 \\
Myo-1 & 25 & 27.6140 & 110.5 \\
Myo-2 & 50 & 54.1840 & 108.4 \\
Myo-3 & 100 & 98.1480 & 98.1 \\
CK-MB-1 & 2.5 & 2.7910 & 111.6 \\
CK-MB-2 & 5 & 5.4130 & 108.3 \\
CK-MB-3 & 10 & 9.0440 & 90.4 \\
\hline
\end{tabular}

